\title{
VALIDATION OF DATA ANALYSIS ROUTINES FOR A THERMAL PROBE APPARATUS USING NUMERICAL DATA SETS
}

Pieter de Wilde*, Richard Griffiths and Steve Goodhew

School of Engineering, Environmental Building Group, University of Plymouth Reynolds Building, Drake Circus, Plymouth

Devon PL4 8AA

United Kingdom

Email: pieter.dewilde@plymouth.ac.uk

Tel: + 44 (0) 1752233695

Fax: + $44(0) 1752232638$

* corresponding author

\section{ABSTRACT}

Most thermal properties of construction materials used in the analysis of building performance have been measured under laboratory conditions, using a guarded hot box or hot plate apparatus. As a consequence, these properties seldom reflect the impact of actual conditions (especially moisture content) on the values of conductivity and diffusivity. Hence there is a need to develop techniques that allow to take into account local conditions, and measure building material properties in situ. One option available is the use of a thermal probe. The thermal probe technique is based on creating a line source in a material sample, and measuring the temperature rise in the sample in reaction to heat being applied. Obviously the data analysis routines used to calculate thermal conductivity and thermal diffusivity based on the temperature rise observed are crucial to the success of the technique.

Transient thermal simulation of a of a model representing a line source in an infinite material sample has been used to generate a set of numerical data sets to validate analysis routines in conjunction with an experimental thermal probe apparatus. Findings show that by careful application of these routines, a close agreement with simulation input values can be achieved, 
with errors of less than one percent. This validates the analysis routines and provides a deeper appreciation of the theoretical behaviour of a thermal probe.

\section{$\underline{\text { KEYWORDS }}$}

Validation, thermal probe, measurement, analysis routines, transient simulation

\section{LIST OF SYMBOLS}

$\alpha \quad$ thermal diffusivity of the specimen $\left[\mathrm{m}^{2} \mathrm{~s}^{-1}\right]$

$\lambda \quad$ thermal conductivity of the specimen $\left[\mathrm{Wm}^{-1} \mathrm{~K}^{-1}\right]$

$\vartheta \quad$ temperature of the thermal probe $[\mathrm{K}]$

$m \quad$ mass per unit length of the thermal probe $\left[\mathrm{kgm}^{-1}\right]$

$c_{p} \quad$ specific heat capacity of the thermal probe $\left[\mathrm{Jkg}^{-1} \mathrm{~K}^{-1}\right]$

$r_{0} \quad$ external radius of the themal probe $[\mathrm{m}]$

$r_{1} \quad$ internal radius of the thermal probe $[\mathrm{m}]$

$\alpha_{\mathrm{p}} \quad$ thermal diffusivity of the thermal probe material $\left[\mathrm{m}^{2} \mathrm{~s}^{-1}\right]$

$\mathrm{H} \quad$ "outer conductivity" of the thermal probe $\left[\mathrm{Wm}^{-2} \mathrm{~K}^{-1}\right]$

$\mathrm{L} \quad$ length of the thermal probe $[\mathrm{m}]$

Q heat supplied per unit length of the thermal probe $\left[\mathrm{Wm}^{-1}\right]$

$\mathrm{T} \quad$ elapsed time of the measurements [s]

$\mathrm{W}$ rate of energy arrival, where $\mathrm{W}=\mathrm{Ql},[\mathrm{W}]$

\section{INTRODUCTION TO THE THERMAL PROBE TECHNIQUE}

Reliable data concerning the thermal properties of building materials, in particular the thermal conductivity $(\lambda)$ and thermal diffusivity $(\alpha)$, is needed for the proper simulation of the thermal behavior of buildings, whether in a design stage, refurbishment project, or research context. However, much of the data currently used in building performance simulation is obtained 
under laboratory conditions, which results in two main problems. Firstly, material properties established using techniques like the guarded hot box method might reflect a dried-out condition, which differs from material properties in actual use. Secondly, actual material properties might be hard to obtain from a handbook due to the multitude of variants of any material, for instance resulting from different manufacturing processes and differences in ingredients.

An alternative to drawing on laboratory data sources is the use of in-situ measurements, allowing the capture of actual thermal properties of materials in buildings in use. One technique for measuring such data on site is the use of a thermal probe apparatus. Thermal probes have been developed and used in other industries, such as geotechnics, food and plastic manufacturing; they are only recently being applied to buildings.

Many authors have researched thermal measurement techniques based on transient hot wire/probe techniques. Schleiermacher (1888) first suggested and carried out measurements followed by Niven (1905) who carried out experiments with a platinum wire acting as a line source. Stalhane and Pyk (1931) developed a technique which cased the hot wire within a tube and used a mercury thermometer to record the temperature rise of the tube. Following the mathematical proofs contained in the seminal work, Carslaw and Jaeger (1947,) van der Held and van Drunen (1949) outlined the basis for 'non-stationary', or transient, thermal conductivity measurement. Their technique recorded the temperature at the midpoint of the heating wire and plotted the temperature rise $\Delta \theta$ against the natural logarithm of time (t), the thermal conductivity being deduced from the slope of this straight line $\Delta \theta / \ln (\mathrm{t})$. Hooper and Lepper (1950) recognised the limitations of the guarded hot plate method of measuring thermal conductivity in their investigation concerning the thermal properties of moist soils. 
Not only did moisture migration have to be contended with but the physical structure of the soils had to be disturbed to prepare samples, problems previously recognised by Patten (1909). Blackwell and Misener (1951) studied the methodology developed by Hooper and Lepper and deduced that the contact resistance between the probe and sample was significant. Blackwell (1954) commented upon a number of issues concerning the thermal conductivity probe, regarding heat losses through thermocouple wires affecting temperature measurements, short time measurements and theoretical error levels for various probe length to diameter ratios. De Vries and Peck (1958) developed the work of van der Held, Jaeger and Blackwell and concluded that thermal conductivity values for soils and materials with similar thermal properties could be measured to within $5 \%$ of the accepted values of the thermal properties of these materials. Blackwell (1954) further offered a mathematical solution which potentially allowed the calculation of the thermal diffusivity $(\alpha)$ of any material that could be measured with a known thermal contact resistance $(\mathrm{H})$ between probe and material. Vos (1955) identified various causes for deviation from the linear including thermal capacity, reflection and the effect of inhomogeneity. Niovichenok and Pikus (1975) used an uncased hot wire to measure both the thermal conductivity and diffusivity of various oils, including petroleum jelly. They recognised errors were caused by the limiting factors in their use of Blackwell's solution. Davis and Downs (1980) carried out a critical review of the hot wire transient line source method, as applied to the measurement of thermal conductivity in insulating refractory bricks with thermal conductivities in the region of $0.12-0.6 \mathrm{Wm}^{-1} \mathrm{~K}^{-1}$. Batty et al (1984a), (1984b) carried out a review of the thermal probe technique and performed numerous trial measurements. Jones (1988), following van der Held and van Drunen and Batty, worked on obtaining thermal conductivity results from $\Delta \theta / \ln (\mathrm{t})$ at early times, before linearity had necessarily been reached, by finite element analysis. Campbell et al (1991) used two parallel probes to measure thermal diffusivity in various soils, in a similar method to that used by 
Drury (1988) and Morabito (1989). Davies et al (2004), used dual thermal probes to measure relative and fluctuating moisture content of building envelopes, assuming that the volumetric heat capacity of soil could be measured within $1 \%$ of accepted values when using typical probe geometries and heating times. Moisture content could then be calculated either by comparing results with those of a dry sample or with values from accepted references. Banaszkiewicz et al (1997), following Seiferlin et al (1996) and citing prior successes by de Groot et al (1974) in gases, Sandberg et al (1977) in liquids, Buettner (1955) and Seiferlin et al (1996) in solids, followed traditional line source models. Algorithms were developed whereby, rather than using just two points as a linear asymptote of $\Delta \theta / \ln (\mathrm{t})$, a least squares optimisation process was carried out.

The authors of this article have been developing the thermal probe technique for a number of years (Goodhew, 2000; Goodhew and Griffiths, 2003; Goodhew and Griffiths, 2004), (Pilkington, 2008) following on the work by Batty et al (1984a, 1984b). Various probes were manufactured using copper tubing of assorted diameters with a variety of fillers, such as epoxy, containing a heater wire and thermocouples, before procuring commercially available Hukseflux TP02 150mm probes, and then the shorter 70mm TP08 probes. An image of the latest experimental thermal probe equipment is presented in figure 1.

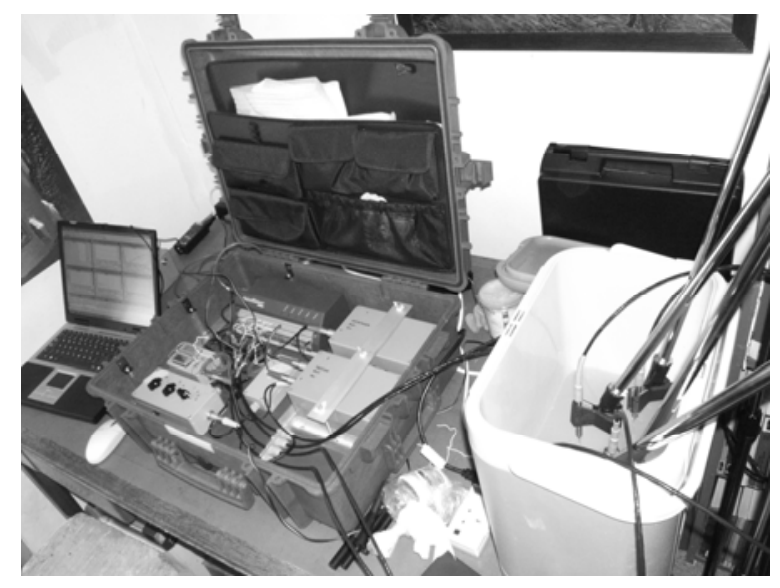


Figure 1. The thermal probe equipment during calibration phase, measuring agar immobilized or stabilized water.

Goodhew (2000), recognised that the probe's outer conductance, or contact resistance between the probe and sample, termed $\mathrm{H}$, was generating a greater influence on results for thermal diffusivity than many previous researchers had accounted for. An iterative optimization routine using the MS Excel add-in program Solver (2007) was developed, in line with Batty's arrangement of Blackwell's work. Goodhew and Griffiths (2003) discussed the appropriate time section of $\Delta \theta / \ln (\mathrm{t})$ for analysis using the Solver routines. Thermal conductivity was found via a regression analysis of the earliest available part of a linear asymptote, before the effects of 'thermal drift' became significant. Goodhew and Griffiths (2004), (2005) reported thermal conductivity, thermal diffusivity and volumetric heat capacity values for mineral oil, magna, paraffin wax and PTFE, measured by thermal probes and using the Solver analysis routines.

For the experimental thermal probe, a probe of length $\mathrm{L}$, radius $\mathrm{r}$ and mass per unit length $\mathrm{m}$ is inserted into a material with thermal conductivity $\lambda$, diffusivity $\alpha$ and density $\rho$. The probe sample combination is allowed to reach thermal equilibrium. A constant power per unit length Q Watts/m is applied to an axially mounted heater within the probe and the probe temperature rise is $\Delta \theta$ after an elapsed heating time $\mathrm{t}$ seconds. This rise in probe temperature is recorded and a graph of the rise is plotted against the natural logarithm of the elapsed time. Blackwell (1954) gave a series expansion for the rise in temperature $\Delta \theta$ at the surface of a probe as a function of time $\mathrm{t}$ :

$$
\Delta \theta=\mathrm{A}\left\{\ln \mathrm{t}+\mathrm{B}+(1 / \mathrm{t})[\mathrm{Clnt}+\mathrm{D}]+\left(1 / \mathrm{t}^{2}\right)[\mathrm{F} \ln \mathrm{t}+\mathrm{G}]+\ldots\right\}
$$


After some time the terms in $\left(1 / \mathrm{t}^{2}\right)$ in this equation are ignored, and the first four terms give $\Delta \theta(4)$ as:

$$
\Delta \theta(4)=\quad \mathrm{A}\{\operatorname{lnt}+\mathrm{B}+(1 / \mathrm{t})[\mathrm{Clnt}+\mathrm{D}]\}
$$

At even longer times the terms in $(1 / \mathrm{t})$ can also be ignored, and the first two terms give:

$$
\Delta \theta(2)=\quad \mathrm{A}(\ln \mathrm{t}+\mathrm{B})
$$

where

$$
\begin{aligned}
\mathrm{A} & =\mathrm{Q} / 4 \pi \lambda \\
\mathrm{B} & =\ln \left(4 \alpha / \mathrm{r}^{2}\right)-\gamma+2 \lambda / \mathrm{rH} \\
\mathrm{C} & =\left(\mathrm{r}^{2} / 2 \alpha\right)\left[1-\alpha \mathrm{mc}_{\mathrm{p}} / \pi \mathrm{r}^{2} \mathrm{~L} \lambda\right] \quad \text { and } \\
\mathrm{D} & =\left(\mathrm{r}^{2} / 2 \alpha\right)\left[\ln \left(4 \alpha / \mathrm{r}^{2}\right)-\gamma+1-\mathrm{B} \alpha \mathrm{mc}_{\mathrm{p}} / \pi \mathrm{r}^{2} \mathrm{~L} \lambda\right]
\end{aligned}
$$

Here $\gamma$ is the Euler constant $0.5772, \mathrm{H}$ is the probe to sample thermal conductance, and $\mathrm{c}_{\mathrm{p}}$ the probe specific heat capacity. The graph of the rise in probe temperature versus the natural logarithm of the elapsed heating time will be a straight line of slope S and intercept $\mathrm{I}$, so that by comparison with equation 2 :

$$
\begin{array}{llll}
\lambda & = & \mathrm{Q} /(4 \pi \mathrm{S}) & \text { and } \\
\alpha & = & \left(\mathrm{r}^{2} / 4\right) * \exp [(\mathrm{I} / \mathrm{S})+\gamma-2 \lambda / \mathrm{rH}]
\end{array}
$$

Note that if the thermal contact between the probe and the sample is perfect then $\mathrm{H}$ is infinite and the term $2 \lambda / \mathrm{rH}$ is zero.

The volumetric heat capacity of the sample $\rho \mathrm{C}$ is given by the ratio of the thermal conductivity to the diffusivity, or $\rho \mathrm{C}=\lambda / \alpha$. For further discussion of the Blackwell expressions see Goodhew and Griffiths (2004), and Batty et al (1984). 
When analysing probe data for each and every power per $\mathrm{m}$, and probe - sample combination, a fundamental question is that of which time series is to used. Or, in other words: to determine when the Blackwell (1954) two constants expression be safely applied to the collected data.

A Solver routine employing the Blackwell four constants expression is used to determine approximate values of sample conductivity and diffusivity, mainly as a guide, but more importantly to determine the elapsed heating time required so that the error between the Blackwell two constants approximation and the Blackwell four constants approximation is less than $1 \%$. The Solver routines were constructed using the readily available Microsoft Excel Solver tool which uses the Generalized Reduced Gradient (GRG2) nonlinear optimization code developed by Leon Lasdon, University of Texas at Austin, and Allan Waren, Cleveland State University (Lasdon et al, 1978) Since the Blackwell expressions contain individual terms with both conductivity and diffusivity a non-linear optimization code was necessary. These Solver routines were developed, by Goodhew and Griffiths (2004), to analyze the practical probe data, which was assumed to have three unknowns, namely (1) the sample thermal conductivity, (2) the thermal diffusivity, and (3) the probe to sample conductance $\mathrm{H}$, which was assumed to be finite. Theoretical probe temperature rises with time for a given probe and sample combination were calculated using both the Blackwell two constant model, $\Delta \theta$ (2), using $\mathrm{A}$ and $\mathrm{B}$ from equation 3 above, and the Blackwell four constant model, $\Delta \theta$ (4) using $\mathrm{A}, \mathrm{B}, \mathrm{C}$ and $\mathrm{D}$ from equation 2 above. The two Solver routines were called Solver 2.3, a routine based upon the Blackwell approximation with two constants and with the three unknowns, and Solver 4.3 a routine based upon the Blackwell approximation with four constants and again the same three unknowns. For every second the difference between the experimental probe rise temperature and the theoretical probe rise temperature is determined, the difference is squared to remove the negative contributions, and 
the squared differences summed. Using an iterative process Solver then finds the minimum of this sum and reports the optimum values of the sample conductivity, the diffusivity and the probe conductance. It is still necessary to know at what time the data may be safely analyzed so that the assumptions is valid.

In this article the model discussed is theoretical in nature and therefore assumes a close to infinite probe conductance and therefore doesn't require the third unknown within the four constant Solver routine. Thus a Solver 4.2 rather than a Solver 4.3 routine is employed to analyse the data from the model.

\section{THE NEED FOR VALIDATION OF THE SOLVER ANALYSIS ROUTINES}

The thermal probe equipment described in the previous section has been used to study a large number of material samples in both laboratory conditions as well as in in field experiments on real buildings (Pilkington et al, 2007; Pilkington, 2008). Initial analysis of the results suggests that the conductivity values can mostly be identified with an accuracy of within $\pm 10 \%$. However, obtaining values for the thermal diffusivity proves more challenging. Moreover, the number of possible practical measurements of the thermal properties of real materials is limited due to constraints of numbers of suitable sample materials that exhibit properties that lend themselves to being appropriate for the probe to measure. The lack of appropriate reference data specific to some materials and the amount of cumulative time required are also limiting factors when undertaking either in situ or laboratory measurements upon materials that have a wide range of thermal properties.

Different factors can have an impact of the accuracy obtained in the experiments. The measured data can be expected to be influenced by a number of physical factors like the contact resistance between the probe and the sample, and heat losses at either end of the 
probe. Findings can also be influenced by the measurement procedure, for instance the value of heating power provided to the probe. Furthermore, validation of the data analysis techniques (the Solver 2.3 and Solver 4.3 routines) is crucial. It is acknowledged by Goodhew and Griffith (2004) that they might need further improvement, especially regarding the identification and selection of a suitable time window in which to determine the slope $\Delta \theta / \ln (\mathrm{t})$

Consequently, the objective of the work described in this article is the use of a thermal simulation model in for the validation of the data analysis routines. Studies with the thermal model provide the opportunity to study the thermal probe from a theoretical point of view, allowing a rigorous testing of the assumptions, mathematics and the data analysis procedures. They allow a comparison of the effects of individual factors one by one, studying their impact on the practical results obtained, something which is difficult in real experiments. Furthermore the use of simulation allows the research to venture into 'extreme' situations, thereby improving the understanding of the theoretical interaction between thermal conductivity, thermal diffusivity, and various temperature rises employed. The advantage here is that a simulation model can have hypothetical conductivity and diffusivity values that are not readily found in real materials.

\section{TRANSIENT SIMULATION FOR THE GENERATION OF NUMERICAL DATA SETS}

The work described in this article addresses the simulation of an infinitely thin and long line heat source in an almost infinite homogenous block of material. Numerical results thereby represent a theoretically very small diameter line heat source, and do not show any effects related to presence of a real probe. For the study, use has been made of the Physibel program 
Voltra that allows calculation of transient heat transfer using the energy balance technique (Physibel, 2005).

Within Voltra, a model has been built of very large slice of a material sample with outer dimensions of $2400 \times 2400 \mathrm{~mm}$, but with a thickness of only $1 \mathrm{~mm}$. The boundary conditions on both faces of this slice of material have been defined as adiabatic, rendering the material infinite in the direction perpendicular to these faces. The line heat source is modeled at the middle of this slice, again perpendicular to the faces, positioned at $1200 \mathrm{~mm}$ from the boundaries of the sample. Voltra uses a rectangular grid, and gridlines have been put at $1 \mathrm{~mm}$ apart closer to the line source in order to approximate the radial distribution of heat, gradually widening the grid further away reaching $20 \mathrm{~mm}$ spacing at the outer edges, see figure 2 . For this model, various combinations of thermal conductivity $\lambda$ and thermal diffusivity $\alpha$ have been entered, with homogenous properties across the whole sample. The application of power at the heart of the line source models an infinitesimal thin probe without any contact resistance.

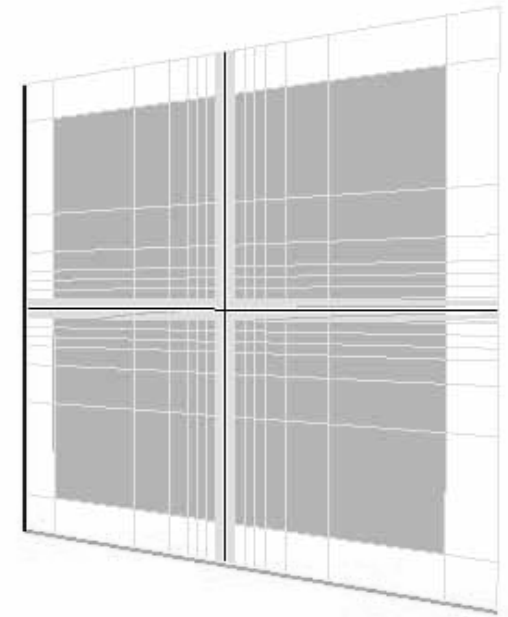


Figure 2. The material sample model in Voltra. The line source is simulated at the origin, perpendicular to the (adiabatic) face of the sample.

Each simulation experiment starts under steady state conditions, where the material sample is at $20.0^{\circ} \mathrm{C}$ and sits in an environment of the same temperature. After an initial 60 seconds (for acclimatisation) a step function is then used to apply a constant power of $3 \mathrm{~W} / \mathrm{m}$ to the line source. The temperatures are monitored at distances of 1, 2, 5, 10 and $50 \mathrm{~mm}$ away from the line source. Simulation results then have been exported to Microsoft Excel, where the first 60 seconds (steady state situation at $20^{\circ} \mathrm{C}$ ) has been discarded and the temperature rise transformed to data for $\Delta \theta / \ln (\mathrm{t})$. Regression analysis is then carried out, and the thermal conductivity $\lambda$ and thermal diffusivity $\alpha$ calculated using equations (4) and (5). The volumetric heat capacity (VHC) or $\rho \mathrm{C}$ is given by the ratio $\lambda / \alpha$.

\section{APPLICATION OF SOLVER ANALYSIS ROUTINES TO NUMERICAL DATA SETS}

Numerical data sets have been generated in Voltra for two main categories: typical construction materials and theoretical materials. For the typical construction materials, properties for $\lambda, \rho$ and $\mathrm{C}$ have been taken from the ASHRAE Handbook of Fundamentals (2005). Subsequently a range of theoretical materials has been studied, varying values of $\lambda$ and $\rho \mathrm{C}$ over a wide domain, allowing assessment over the whole range and including very extreme cases, in order to see how these impact on results obtained.

\section{TYPICAL CONSTRUCTION MATERIALS}

Table 1 and 2 show the results of some of the simulation studies for the typical construction materials. All the studies were conducted with a heater power of $3 \mathrm{~W} / \mathrm{m}$ and the temperatures 
were recorded at $1 \mathrm{~mm}$ from the simulated axial heater. Table 1 gives the model input data, together with the Solver 4.3 error time for $\mathrm{E} \%=1, \mathrm{t}_{\mathrm{e}}$ seconds. These materials consisted of three mineral samples and three vegetable samples. The input data for these six real samples was taken from the ASHRAE Handbook of Fundamentals (2005). The Solver 4.3 routine was used to determine the values of $t_{e}$ and approximate values for the sample thermal conductivity and diffusivity. Table 2 shows the results, the first column giving the time interval chosen for the regression analysis, the final column the volumetric heat capacity of the samples. As can be seen from comparing Table 1 and Table 2 there is reasonable agreement between the input thermal data and the values obtained from the analysis routine. As the temperature measurements were made to two decimal places, when the rise temperature was only $1 \mathrm{C}$ over the time interval then the results would be expected to be uncertain by about $1 \%$. This uncertainty becomes more obvious in the values of the diffusivity as this quantity depends on the intercept on the temperature axis. This is seen in the resulting values of the diffusivity when the output values are compared with the input. 
Table 1. Voltra simulation input data

\begin{tabular}{|c|c|c|c|c|c|}
\hline Material & $\begin{array}{l}\text { Density } \\
{\left[\mathrm{kg} / \mathrm{m}^{3}\right]}\end{array}$ & $\begin{array}{c}\text { Conductivity } \\
{[\mathrm{W} / \mathrm{mK}]}\end{array}$ & $\begin{array}{l}\text { Diffusivity } \\
{\left[{ }^{*} 10^{7} \mathrm{~m}^{2} / \mathrm{s}\right]}\end{array}$ & $\begin{array}{c}\text { Specific } \\
\text { heat capacity } \\
{[\mathrm{J} / \mathrm{kgK}]}\end{array}$ & $\begin{array}{c}\text { Solver } 4.2 \\
\text { error time } t_{\mathrm{e}} \\
{[\mathrm{s}]}\end{array}$ \\
\hline Fire clay brick & 1790 & 1.00 & 6.73 & 830 & 80 \\
\hline Brick & 1970 & 0.7 & 4.44 & 800 & 110 \\
\hline Sand & 1520 & 0.33 & 2.71 & 800 & 260 \\
\hline Oak & 750 & 0.18 & 1.00 & 2390 & 830 \\
\hline Fir & 540 & 0.12 & 1.84 & 1210 & 420 \\
\hline Cellulose & 54 & 0.057 & 8.12 & 1300 & 80 \\
\hline
\end{tabular}

Table 2. Results from regression analysis (using Solver routines) of Voltra simulation output

\begin{tabular}{|l|c|c|c|c|c|}
\hline Material & $\begin{array}{c}\text { Interval for } \\
\text { analysis } \\
{[\mathrm{s}]}\end{array}$ & $\begin{array}{c}\text { Conductivity } \\
{[\mathrm{W} / \mathrm{mK}]}\end{array}$ & $\begin{array}{c}\text { Diffusivity } \\
{\left[* 10^{7} \mathrm{~m}^{2} / \mathrm{s}\right]}\end{array}$ & $\begin{array}{c}\text { Specific } \\
\text { heat capacity } \\
{[\mathrm{J} / \mathrm{kgK}]}\end{array}$ & $\begin{array}{c}\text { Volumetric } \\
\text { heat capacity } \\
{\left[\mathrm{MJ} / \mathrm{m}^{3} \mathrm{~K}\right]}\end{array}$ \\
\hline Fire clay brick & 100 to 300 & 1.00 & 6.31 & 880 & 1.58 \\
\hline Brick & 150 to 350 & 0.7 & 4.16 & 850 & 1.68 \\
\hline Sand & 300 to 500 & 0.33 & 2.57 & 840 & 1.28 \\
\hline Oak & 1300 to 1500 & 0.18 & 0.98 & 2400 & 1.81 \\
\hline Fir & 500 to 1500 & 0.12 & 1.9 & 1190 & 0.64 \\
\hline Cellulose & 100 to 500 & 0.058 & 8.36 & 1320 & 0.07 \\
\hline
\end{tabular}

\section{THEORETICAL MATERIALS}

In order to get a better understanding of the impact of input parameters on computational outcomes, a set of theoretical materials have been studied, varying the inputs of $\lambda$ and $\rho C$. The following combinations have been reviewed, see table 3 . 
Table 3. The three combination of the pairings of magnitude of $\lambda$ and $\rho C$

\begin{tabular}{|l|l|l|}
\hline low $\lambda$ & average $\lambda$ & high $\lambda$ \\
low $\rho \mathrm{C}$ & low $\rho \mathrm{C}$ & low $\rho \mathrm{C}$ \\
\hline low $\lambda$ & average $\lambda$ & high $\lambda$ \\
average $\rho \mathrm{C}$ & average $\rho \mathrm{C}$ & average $\rho \mathrm{C}$ \\
\hline low $\lambda$ & average $\lambda$ & high $\lambda$ \\
high $\rho \mathrm{C}$ & high $\rho \mathrm{C}$ & high $\rho \mathrm{C}$ \\
\hline
\end{tabular}

The input for the Voltra simulations as shown in table 3 has been implemented by $\lambda$ values of 0.01 (low), 0.6 (average) and 2.0 (high). Values for $\rho \mathrm{C}$ have been varied by changing $\mathrm{C}$ only, with $\rho$ taken to be constant at 1000 . Values used for $\mathrm{C}$ are 100 (low $\rho \mathrm{C}$ ), 2000 (average $\rho \mathrm{C}$ ) and 6000 (high $\rho C$ ). Note that "low", "average" and "high" values are related to common materials found in building construction. Most of the Voltra studies showed the characteristic behaviour observed in previous measurements, (Goodhew and Griffiths, 2004) when the rise in probe temperature was plotted against the natural logarithm of the elapsed heating time. Values of the calculated thermal conductivity from the Voltra simulations generally agreed with the values inputted to the model. However, with the low thermal conductivity study, with conductivity as $0.01 \mathrm{~W} / \mathrm{m}^{2} \mathrm{~K}$, and for the three associated values of the specific heat capacity, $(100,2000$ and $6000 \mathrm{~J} / \mathrm{kgK})$, and with the adopted heating input of $3 \mathrm{~W} / \mathrm{m}$, the rises in temperature at $1 \mathrm{~mm}$ from the heater were large compared with the laboratory studies. The Voltra model showed typical temperature rises of 40 to $100^{\circ} \mathrm{C}$ in $200 \mathrm{~s}$, whereas in the laboratory or field studies 6 to $9^{\circ} \mathrm{C}$ would be expected or used. To analyse the low thermal conductivity data from the model studies provided an interesting vehicle for exploring the analysis routine of Goodhew and Griffiths (2004). From the physical data it would be 
expected that the rise would take a long time, small conductivity, but the magnitude of the rise in temperature would be large for materials with low specific heat capacity.

There are practical reasons for restricting the data analysis to 300 to $400 \mathrm{~s}$ when analysing data from the laboratory and field. For example, when the increase in the rise in probe temperature becomes too small for practical power inputs, there is considerable data scatter and axial heat losses can depending upon the sample material being measured, mar the results. However, the Voltra studies consider the heat flow in an infinite medium with no physical probe and therefore the results provide an opportunity to view the thermal probe technique from a simple theoretical stance, and to test the model, the assumptions, the mathematics or theory and the data analysis strategies. As there is no physical probe in the Voltra simulation the probe to sample conductance $\mathrm{H}$ is infinitely large and the thermal capacity of the probe must be zero. This provides the opportunity to rigorously test the analysis routine employing equations 3 and 4 with regression analysis to determine both the thermal conductivity and the diffusivity.

As stated in the introduction, when analysing probe data for each and every power per $\mathrm{m}$, and probe - sample combination, it is crucial to determine when the Blackwell (1954) two constants expression be safely applied to the collected data.

For the application of the Solver routines, the following criterion was suggested by Goodhew and Griffiths (2004) to determine the time interval for the analysis of the data. The \% Error for the rise temperature using $2(\Delta \theta(2))$ or $4(\Delta \theta(4))$ constants, $\mathrm{E} \%$, was defined as:

$$
\mathrm{E} \%=100[\Delta \theta(4)-\Delta \theta(2)] / \Delta \theta(4)
$$

This was taken arbitrarily to be $1 \%$, and the corresponding time $t_{\mathrm{e}}$ can be found from: 


$$
1 \%=100\left\{\left(1 / \mathrm{t}_{\mathrm{e}}\right)\left[\mathrm{Clnt}_{\mathrm{e}}+\mathrm{D}\right] /\left\{\operatorname{lnt}_{\mathrm{e}}+\mathrm{B}+\left(1 / \mathrm{t}_{\mathrm{e}}\right)\left[\operatorname{Clnt}_{\mathrm{e}}+\mathrm{D}\right]\right\}\right.
$$

A graph of $\mathrm{E} \%$ versus time allows the time $t_{e}$ to be determined, or alternatively the "goal seek" routine in Excel may be used to solve the above expression for $t_{\mathrm{e}}$. This allows the appropriate time window for the regression analysis of the data, or the application of Solver 2.3 , to be set. The analysis must start after $t_{\mathrm{e}}$ second has elapsed.

To illustrate this process a detail discussion of the analysis of one set of simulation data will be described. The chosen set of data for this discussion is the Voltra study with thermal conductivity $0.01 \mathrm{~W} / \mathrm{m}^{2} \mathrm{~K}$, thermal capacity $100 \mathrm{~J} / \mathrm{kgK}$, and density $1000 \mathrm{~kg} / \mathrm{m}^{3}$, giving a diffusivity of $10^{-7} \mathrm{~m}^{2} / \mathrm{s}$. The heater power was $3 \mathrm{~W} / \mathrm{m}$, with the temperature rise in the medium measured at $1 \mathrm{~mm}$ from the line source. A graph of the rise in temperature versus the natural logarithm of the elapsed heating time is shown in figure 3. Visual inspection of the curve in figure 3 confirms that there is the characteristic "s" shape before the asymptotic approach to the final straight line beyond natural logarithm time of 5.2, or beyond a time of about $180 \mathrm{~s}$. The temptation is to apply the regression analysis to this data from 30 to $1500 \mathrm{~s}$, or natural logarithm 3 to 7 . Here, the resulting conductivity is $0.0103 \mathrm{~W} / \mathrm{m}^{2} \mathrm{~K}$ and diffusivity $1.12 \times 10^{-7} \mathrm{~m}^{2} / \mathrm{s}$. The conductivity is $3 \%$ above the true or input value, while the diffusivity is $12 \%$ above the input value. These errors are of similar magnitude to those often found in the experimental laboratory and field work.

Following the recipe proposed by Goodhew and Griffiths (2004) a Solver 4.2 routine was applied to this data over the time interval 1300 to 1500 s. Solver 4.2 is a Blackwell 4 constant expression with 2 variables, the sample thermal conductivity and diffusivity. The probe conductance was set to $10^{16} \mathrm{~m}^{2} \mathrm{~K} / \mathrm{W}$ or a large number, arrived at by saying 100 million is large so that squared must be very large. The probe capacity is set to zero. Figure 4 shows the 
main graph from the Solver sheet. Here the Solver line describes the data only at times greater than 400 to $500 \mathrm{~s}$. The Solver 4.2 routine gave low values of conductivity and diffusivity, but the interesting feature here is that the time that must elapse before the error between the 2 and 4 constant expressions falls to below $1 \%$ is 780 s, as can be seen in figure 5 the theoretical 0.01 curve. Therefore, the conclusion to be drawn is that the equations 4 and 5 cannot be applied to this data set until the heating time exceeds 800 s.

Applying the regression analysis to the Voltra data over the time interval 850 to $1500 \mathrm{~s}$, results in a thermal conductivity of $0.01002 \mathrm{~W} / \mathrm{m}^{2} \mathrm{~K}(+0.2 \%)$, and diffusivity $0.973 \times 10^{-7} \mathrm{~m}^{2} / \mathrm{s}$ $(-2.7 \%)$. These errors are calculated in terms of the known true values for conductivity and diffusivity that were initially inputted into the Voltra package. With the Solver 2.2 routine, (Solver 2.2 has the two constants A and B of equation 1 and the two unknowns conductivity $\lambda$ and diffusivity $\alpha$ with the probe to sample conductance $H$ set to a very high number), and the data set in the time window 850 to $1500 \mathrm{~s}$, the resulting conductivity is $0.01002 \mathrm{~W} / \mathrm{m}^{2} \mathrm{~K}$, $(+0.2 \%)$, diffusivity $0.975 \times 10^{-7} \mathrm{~m}^{2} / \mathrm{s}(-2.5 \%)$, again errors in terms of true values.

Finally, moving the Solver 2.2 time window to longer times, namely 1300 to 1500 s, the resulting conductivity is $0.01008 \mathrm{~W} / \mathrm{m}^{2} \mathrm{~K},(+0.8 \%)$, diffusivity $1.01 \times 10^{-7} \mathrm{~m}^{2} / \mathrm{s}(1 \%)$, again errors in terms of true value. When the regression analysis was applied to the data set in the time window 1300 to $1500 \mathrm{~s}$, the resulting conductivity was found to be $0.01007 \mathrm{~W} / \mathrm{m}^{2} \mathrm{~K}$, $(+0.7 \%)$, and diffusivity $1.006 \times 10^{-7} \mathrm{~m}^{2} / \mathrm{s}(0.6 \%)$. This represents close agreement between model, theory and the Voltra simulation. 


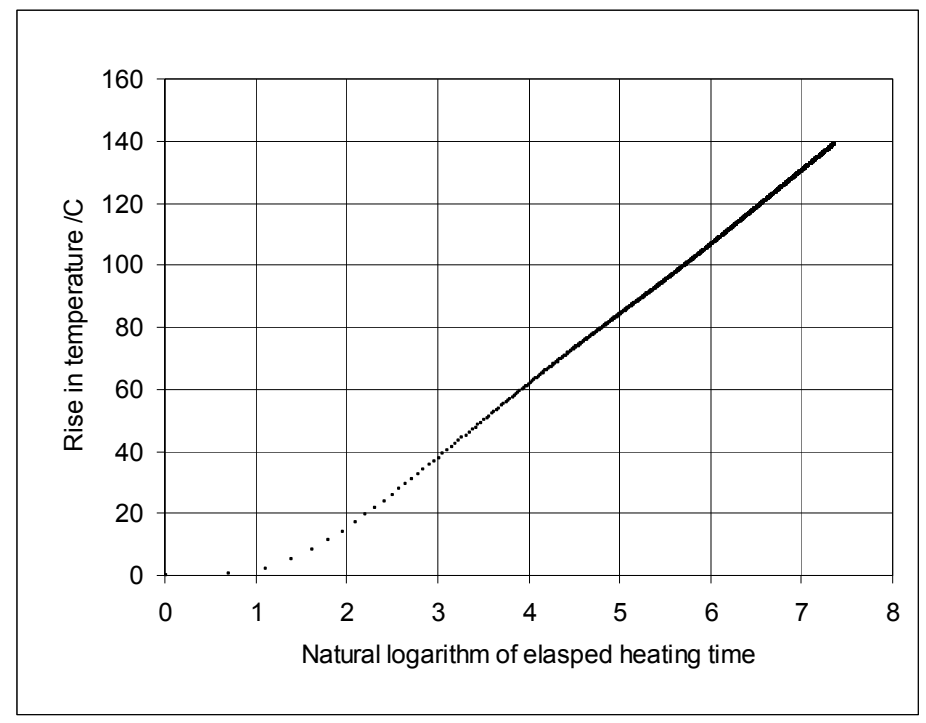

Figure 3: Graph of rise in probe temperature versus natural logarithm of elapsed heating time for the Voltra simulation on a theoretical material with conductivity $0.01 \mathrm{~W} / \mathrm{mK}$, density $1000 \mathrm{~kg} / \mathrm{m}^{3}$, and specific heat capacity $100 \mathrm{~J} / \mathrm{kgK}$. Temperature measured at $1 \mathrm{~mm}$ from line heat source.

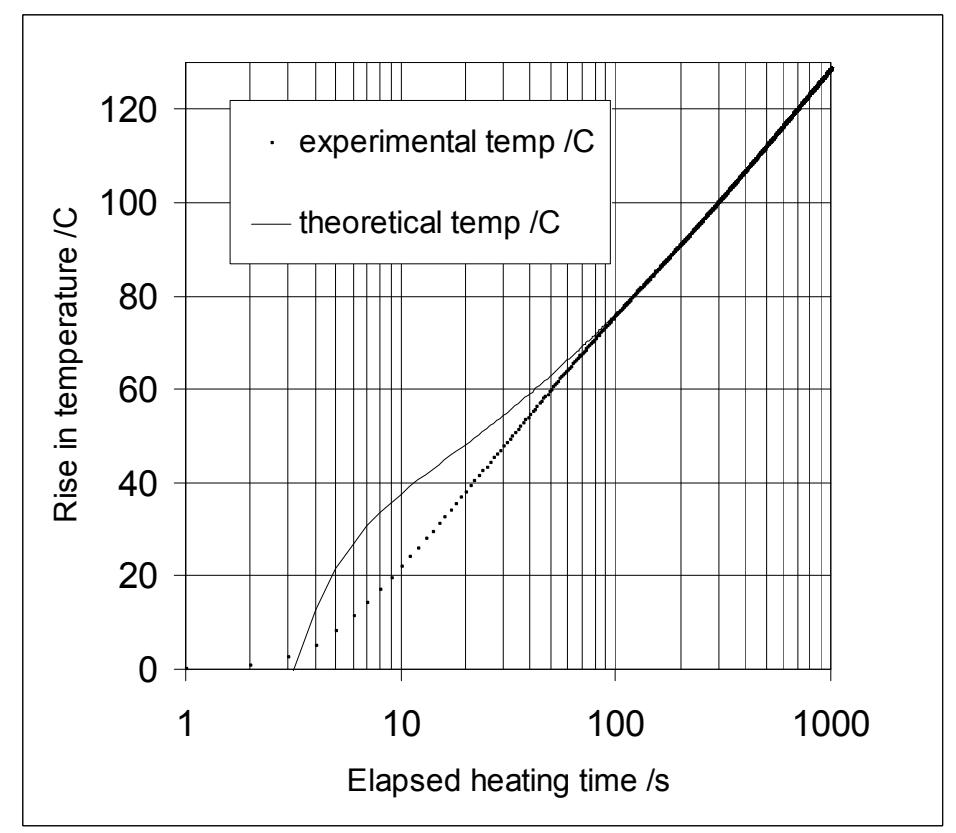

Figure 4: The Solver 4.2 predicted or theoretical temperature rise plotted with the Voltra simulation results, here denoted as experimental temp $/ C$, both as functions of the elapsed heating time in seconds. 


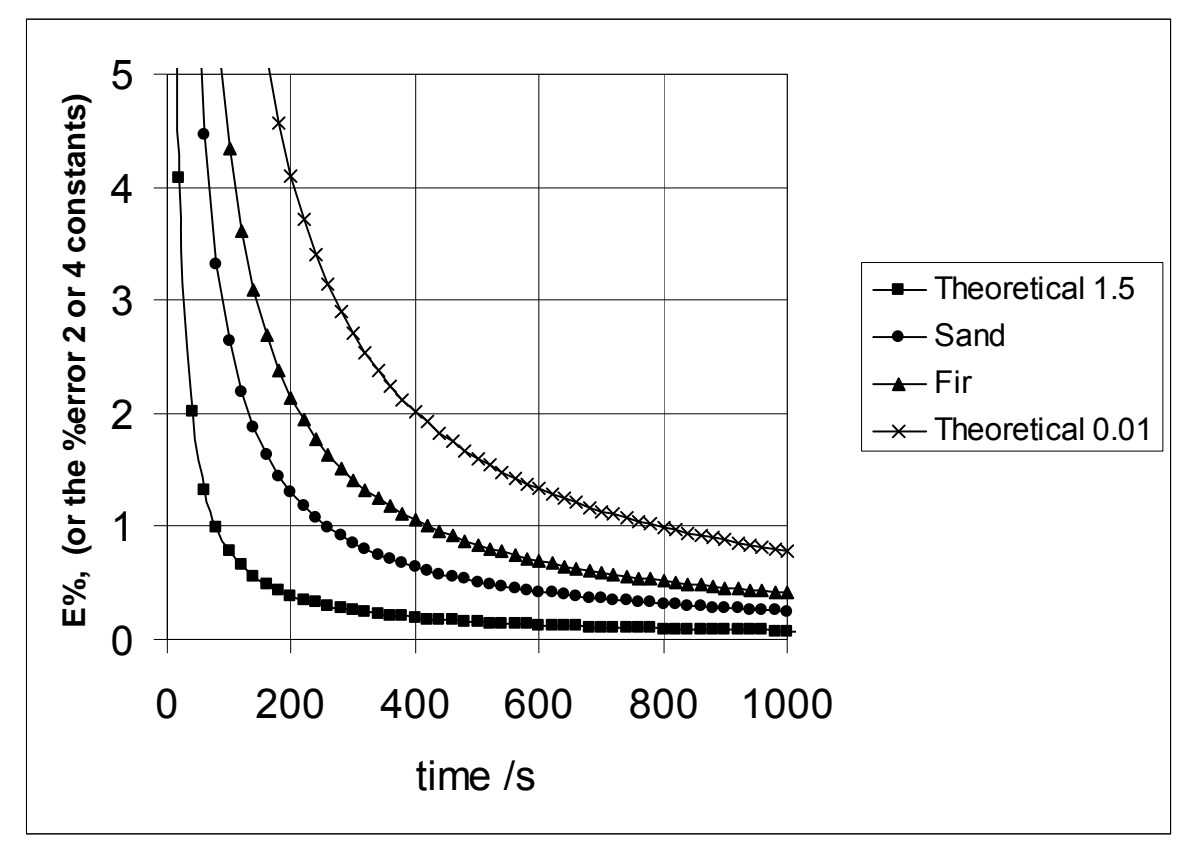

Figure 5: Graph of E\%, or the \%error 2 or 4 constants plotted as a function of time for the theoretical materials with conductivities 1.5 and $0.01 \mathrm{~W} / \mathrm{mK}$, and for the sand and fir simulations. A $1 \%$ error or less is only possible ahter heating times of $70 \mathrm{~s}$ for Theoretical material 1.5, $260 \mathrm{~s}$ for sand, $420 \mathrm{~s}$ for fir and $780 \mathrm{~s}$ for the Theoretical 0.01.

The graph shown in Figure 5 where the input volumetric heat capacity is plotted as a function of the time $t_{e}$, has an impact on the practical application of this probe technique to real materials, both in the field and in the laboratory. Given that the results for oak and cellulose do not follow the trend, as seen in Figure 5 there is a clear curve showing that as the volumetric heat capacity of materials decreases the regression analysis time interval based upon the $t_{e}$ value increases to longer times. The values for oak may be due to the wood being wet, or green, and the value for cellulose confused due to possible heat transfer by radiation and convection within the sample. 
Other materials, from as yet unpublished work on real samples in the laboratory, show a similar pattern, with agar or stabilized water having a volumetric heat capacity of 4.2 $\mathrm{MJ} / \mathrm{m} 3 \mathrm{~K}$ and corresponding $\mathrm{t}_{\mathrm{e}}$ of 9 seconds, for cob (earth walling in the West Country, UK) 1.2 MJ/m3K, 65s, and Celcon concrete block $0.63 \mathrm{MJ} / \mathrm{m} 3 \mathrm{~K}, 260 \mathrm{~s}$. These three values of te were calculated with a finite probe conductance $\mathrm{H}$ of $650 \mathrm{~W} / \mathrm{m} 2 \mathrm{~K}$. The practical problem arises due to axial conduction along the probe and therefore measurements need to be made quickly and as near to the start as possible. For low volumetric heat capacity materials the assumption that long times have been reached to satisfy the Blackwell equations may not be possible without errors in the probe temperature rises caused by axial heat losses.

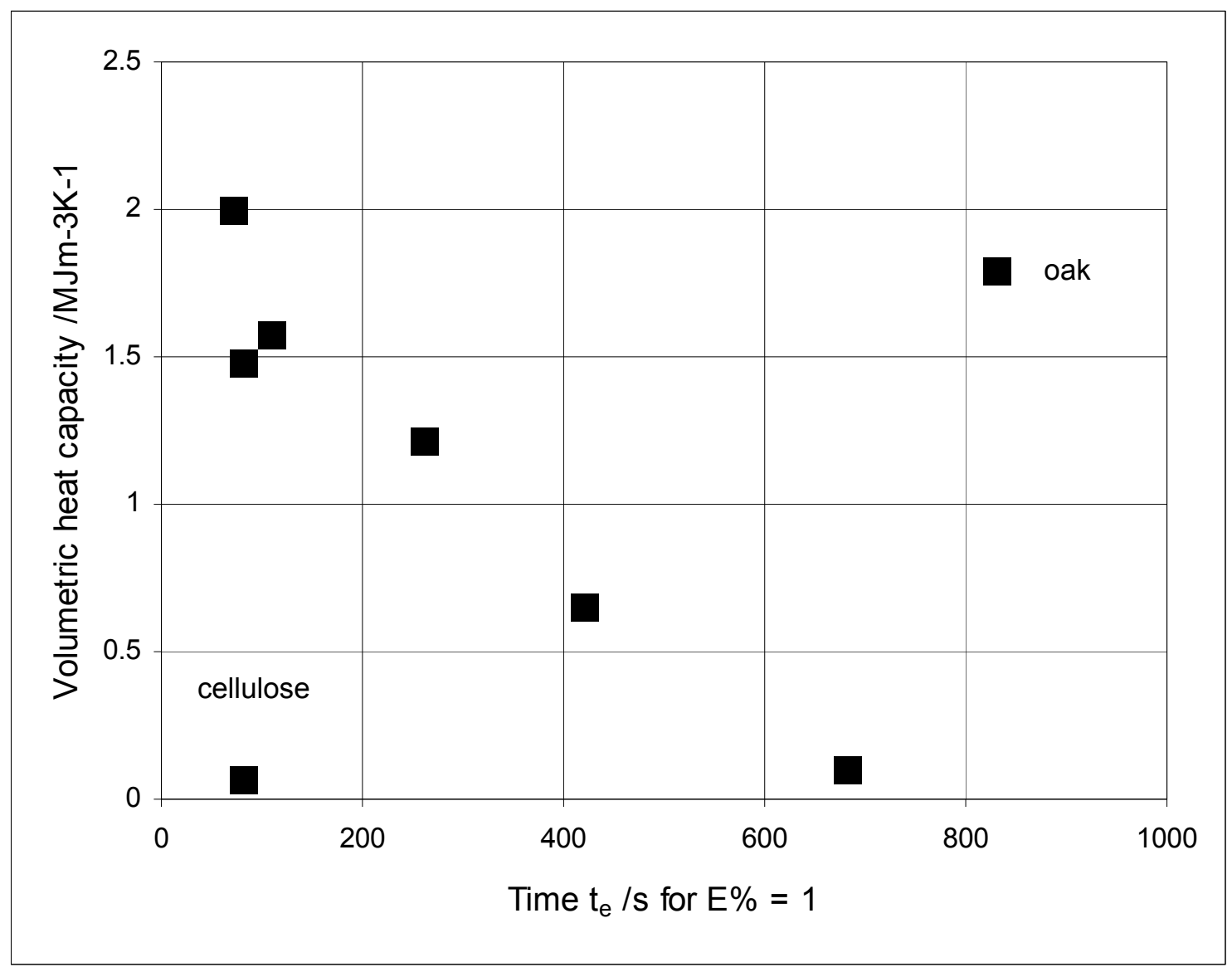

Figure 5: Graph of sample volumetric heat capacity against the E\% $=1 \%$ times $t_{e}$. 


\section{CONCLUSIONS AND REMARKS}

This article describes the use of transient thermal simulation to validate analysis routines used

to process measurements conducted with an experimental thermal probe apparatus. The following conclusions have been drawn from the work described:

1. Using the transient heat transfer program Voltra, a line source in an (almost) infinite material sample has been modeled. The simulation model has been used to generate datasets which have been analyzed for $\lambda$ and $\alpha$ by means of the same analysis routines (Solver 4.2, Solver 2.3) that are currently being used to process experimental data from an actual thermal probe apparatus.

2. The Voltra model, the assumptions and the theory provided by Blackwell (1954) appear to be satisfactorily describing the practical arrangements. Discrepancies between input data inserted into the Voltra model and material properties obtained through application of the Solver routines are in the order of $1 \%$, which is in line with the fact that the Voltra model has been set to report temperatures with two decimal places only.

3. The importance of selecting the appropriate power for the experimental work is underlined, since long times also require measurable temperature rises, that is temperature rises that are clearly above the experimental scatter.

4. Applying the regression analysis to data sets at inappropriate time windows, that is short times, often leads to values for the thermal constants that appear of suitable magnitude, but closer and more careful examination of the data can lead to more accurate values for these thermal constants.

5. The Voltra study has validated the analysis routines as proposed by Goodhew and Griffiths (2004) for a simplified model, that is a model with very large probe to sample conductance and zero probe thermal capacity: 
- The Solver 4.3 routine has been demonstrated to provide crucial information needed to select a correct time interval for the application of regression analysis to the measured data, by allowing the error between the Blackwell 2 and 4 constant expressions to be explored.

- The Solver 2.3 routine applied to the correct time window leads to accurate values of both conductivity and diffusivity, which have been demonstrated to have an error of less than $1 \%$.

\section{$\underline{\text { FUTURE WORK }}$}

Further work will be carried out to advance the understanding of the behaviour of an actual thermal probe apparatus. This will involve modeling of a probes with finite dimensions, specific probe materials, and various internal probe configurations, and inclusion of boundary effects for both probe and measurement sample. Practical issues such as the timing of measurements, the use and type of heat-sink materials used to allow good contact between the body of the probe, increasing the probe conductance $(\mathrm{H})$, and the sample materials can be investigated. It is anticipated that through the use of dynamic simulation modeling, the time span needed to further develop the thermal probe technique and it's application to in-situ measurements of the thermal properties of building materials will be considerably shortened.

\section{$\underline{\text { REFERENCES }}$}

ASHRAE. 2005. ASHRAE Handbook of Fundamentals, SI Edition. Atlanta: American Society of Heating, Refrigerating, and Air-Conditioning Engineers.

Banaszkiewicz M, Seiferlin K, Spohn T, Kargl G, Kömle N (1997). A new method for the determination of thermal conductivity and thermal diffusivity from linear heat source measurements, Rev. Sci. Instruments, 68 (11), 4184-4190 
Batty WJ, O'Callaghan PW, Probert SD (1984a). Assessment of the thermal-probe technique for rapid, accurate measurements of effective thermal conductivities, Applied Energy, 16, 83113

Batty WJ, Probert SD, Ball M, O'Callaghan PW (1984b.) Use of the thermal probe technique for the measurement of the apparent thermal conductivities of moist materials, Applied Energy, 18, 301-317

Blackwell JH, Misener AD (1951). Approximate Solution of a Transient Heat Flow Problem, Proc. Phys. Soc. A 64, 1132-1133

Blackwell JH (1954). A transient-flow method for determination of thermal constants of insulating materials in bulk. Part 1-Theory, Journal of Applied Physics, 25 (2), 137-144

Buettner K (1955). Evaluation of soil heat conductivity with cylindrical test bodies, Transactions, American Geophysical Union, 36 (5), 831-837

Campbell GS, Calissendorff C, Williams JH (1991). Probe for measuring soil specific heat using a heat-pulse method, Soil Sci. Soc. Am. J. 55, 291-293

Carslaw HS, Jaeger JC (1947). Conduction of heat in solids, University Press, Oxford.

Davis WR, Downs A (1980). The hot wire test - a critical review and comparison with the BS 1902 panel test, Transactions British Ceramic Society, 79, 44-52

Drury MJ (1988. A simple needle-probe method for measuring thermal diffusivity of unconsolidated materials, Geothermics, 17, (5-6), 757-763

Goodhew SM (2000). The thermal properties of cob buildings of Devon, PhD Thesis. University of Plymouth (unpublished)

Goodhew SM, Griffiths R (2003). Handbook giving practical details for thermal probe studies with iterative methods for data analysis using Solver routines, University of Plymouth, Joint Schools of the Built Environment, internal report, unpublished

Goodhew SM, Griffiths R (2004). Analysis of thermal probe measurements using an iterative method to give sample conductivity and diffusivity data, Applied Energy, 77, 205-224

Goodhew SM, Griffiths R (2005). Sustainable walls to meet the building regulations, Energy and Buildings, 37, 451-459

de Groot JJ, Kestin J, Sookiazian H (1974). Instrument to measure the thermal conductivity of gases, Physica, 75, 454-482

Van der Held EFM; Van Drunen FG (1949). $A$ method of measuring the thermal conductivity of liquids. Physica A, 15 (10), 865-881

Hooper FC, Lepper FR (1950). Transient heat flow apparatus for the determination of thermal conductivities, Transactions American Society of Heating and Ventilation Engineers 56, 309324 
Jones BW (1988). Thermal conductivity probe: development of method and application to a coarse granular medium, J. Phys. E: Sci. Instrum. 21, 832-839

Lasdon ,LS Waren ,AD Jain ,A Ratner, M (1978) Design and Testing of a Generalized Reduced Gradient Code for Nonlinear Programming, ACM Transactions on Mathematical Software (TOMS), 4 (1), 34-50.

Niven C (1905). On a Method of Finding the Conductivity for Heat, Proceedings of the Royal Society of London. Series A, Containing Papers of a Mathematical and Physical Character, 76, (507) 34-48

Niovichenok LN, Pikus Yu M (1975). Joint determination of thermal properties by a probe method, Journal of Engineering Physics and Thermophysics 29, .3,1116-1118

Pilkington, B., P. de Wilde, P., S. Goodhew, R. Griffiths, 2006. Development of a Probe for Measuring In-situ the Thermal Properties of Building Materials. Proceedings of PLEA'06, 23rd International Conference, Geneva, Switzerland, September 6-8 2006, 665-670

Pilkington, B., R. Griffiths, S. Goodhew, P. de Wilde, 2007. Thermal probe technology for buildings: the transition from laboratory to field measurements. Article under review for the ASCE Journal of Architectural Engineering

Pilkington, B., 2008. In-Situ Measurements of Building Materials Using a Thermal Probe. PhD Thesis, University of Plymouth, to be submitted in February 2008 (unpublished).

PHYSIBEL. 2005. Voltra and Sectra Manual, version 5.0w. Maldegem: Physibel Software.

Schleiermacher A (1888). On the thermal conduction of gas, Wiedeman Ann. Phys. 34, 623646

Seiferlin K, Komle NI, Kargl G, Spohn T (1996). Line heat-source measurements of the thermal conductivity of porous $\mathrm{H} 2 \mathrm{O}$ ice, $\mathrm{CO} 2$ ice and mineral powders under space conditions, Planet. Space Sci., 44 (7), 691-704

Stalhane B and Pyk S (1931). New method for measuring the thermal conductivity coefficients, Teknisk Tidskrift. 51. 389-96

Vos BH (1955). Measurements of thermal conductivity by a non-steady-state method, Appl. Sci. Res. Section A, 5, 425-438

de Vries DA, Peck AJ (1958). On the cylindrical probe method of measuring thermal conductivity with special reference to soils. 1. Extension of theory and discussion of probe characteristics, Australian Journal of Physics, 11, 255-271 